\title{
A Fuzzy Mathematical Model for Multi Criteria Group Decision Making- An Application in Supply Chain Management
}

\author{
A. Uma Maheswari \\ Quaid-E-Millath Government College for Women \\ Chennai-600002
}

\author{
P. Kumari \\ G.S.S.Jain College for Women, Chennai-600007
}

\begin{abstract}
This paper presents a Fuzzy Multi-Criteria Group Decision analysis approach for selecting supplier in a supply chain management. Supplier selection has attained supreme importance for companies in the current scenario because of increasing global competition. Proper selection of suppliers plays a crucial role on the overall performance of the company. The number of available alternatives in the current market is on a rise and hence it becomes difficult to select a supplier from a large lot. An attempt has been made to develop a new composite model using the Fuzzy method based on Schwartz Sequential Dropping method. Finally, an example based on a case study is given to demonstrate the procedure of the proposed methodology.
\end{abstract}

\section{Keywords}

Supplier Selection, Fuzzy Multi-Criteria Decision Making (FMCDM), Linguistic Variables, Triangular Fuzzy Numbers (TFN).

\section{INTRODUCTION}

Decision Making deals with the process of selecting the best alternative from different available alternatives. Due to complexity in decision making situations, relevant aspects of a decision making problem are solved by several decision makers DM's.

Group decision-making is defined as a decision situation in which there is more than one individual involved. "Groups make better judgments than average individual members in analysis and evaluation tasks" (McGrath, 1984; Nah \& Benbasat, 1999).

The authors who have investigated supplier selection include; Jainet al. (2007), Kannan and Noorul Haq (2007), Sevkli et al. (2007), Chan et al. (2008), Lee and Ou-Yang (2008), Che (2010), Liu and Zhang (2010), Ravindran et al. (2010), Senet al. (2010), Talluri and Lee (2010) and Yao et al. (2010). For a comprehensive account of the evolution of supplier selection criteria and methods, see (Zhang et al. 2003) and (Ho et al. 2010).

Fuzzy Multi-Criteria Decision Making MCGDM deals with the Uncertainity and imprecision associated with the real life situations. DM's may not be able to discriminate clearly the degree to which one alternative is better than another. To model the real life situations crisp data is inadequate. Hence fuzzy sets are used to overcome this difficulty. A common feature among these techniques is how the rankings of the potential suppliers are determined (Ordoobadi,2009) and that most often FMCDM rankings are assigned based on two factors: the importance weight of the attributes and suppliers' performance with respect to these attributes. Also if the decision makers express their preferences in pure numeric scales the subjectivity and imprecision associated with perceptions are lost by forcing the decision makers to use numeric scales. As a result linguistic assessments represented in the form of linguistic variables are often used for adequately modeling the uncertainty and imprecision in the decision making process.

Fuzzy logic methodology [1] allows the decision maker to express preferences in linguistic terms. The subjectivity and vagueness in the supplier selection process is dealt with by using fuzzy triangular numbers TFN's for linguistic terms [2] Incorporated the decision makers_attitude towards preference, a crisp overall performance value is obtained for each alternative based on the concept of Fuzzy Multiple Criteria Group Decision Making (FMCGDM). A case study consisting of four suppliers solicited from Asia Energy CorporationTechnologies Unlimited, a small scale industry located in Chennai, India illustrates the effectiveness of the proposed approach. Using fuzzy membership functions and fuzzy mathematical operators a fuzzy score is determined for each supplier. These fuzzy scores are then converted to crisp values through a defuzzification process to make the ranking of the suppliers a straightforward task. The supplier with the highest ranking is selected.

\section{MULTI CRITERIA MATHEMATICAL METHOD}

Multi Criteria Decision making MCDM is a modeling and a methodological tool of dealing with complex engineering or management problems. DM's often face many problems with incomplete and vague information. Multi criteria decision making refers to select or rank alternatives from available alternatives with respect to multiple, usually conflicting criteria involving either a single decision maker or multiple decision maker[3]. Multi criteria decision making can be classified as (a) multi attribute decision making and (b) multi objective decision making[4,5]. Multi attribute decision making problems are assumed to have a predetermined, limited number of decision alternatives.

Multi attribute decision making involves in the evaluation, selection and ranking of alternatives from available alternatives with respect to various criteria. Multi objective decision making involves the selection of the satisfactory alternative from among a set of alternatives based on the preference information of the decision maker in relation to the priorities of the evaluation criteria and objectives and the relationships between 
the objectives and criteria in consideration (Iz and Jelassi, 1990; Quaddus and Siddique, 1996).

\subsection{FUZZY MULTICRITERIA MATHEMATICAL METHOD}

To effectively represent the subjective and imprecise information inherent in the multi criteria decision making problem, the application of fuzzy sets theory has proven to be effective [1] for adequately modeling the subjectiveness and imprecision, leading to the development of fuzzy multi criteria analysis approaches for solving the multi criteria decision making problem in a fuzzy environment [6]. There are a number of very good surveys on fuzzy MCDM [ 7, 8 ]

\section{SOME PRELIMINARY CONCEPTS}

\subsection{Fuzzy Sets}

A fuzzy set $\mathrm{A}$ of the universe of discourse $\mathrm{U}$ is defined by a membership function $A: U \rightarrow[0,1]$, where $A(x)$ is the degree of membership of $\mathrm{x}$ in $\mathrm{A}$ and $[0,1]$ is the closed unit interval on the real line R. Very often, fuzzy set A of $\mathrm{U}$ can be expressed as

$\mathrm{A}=\left\{\left(\mathrm{x}, \mu_{\mathrm{A}}(\mathrm{x})\right) / \mathrm{x} \varepsilon \mathrm{X}, 0 \leq \mu_{\mathrm{A}}(\mathrm{x}) \leq 1\right\}$.

As a comparison, a classical non-fuzzy set B is usually defined as a binary membership function $\mathrm{B}: \mathrm{U} \rightarrow\{0,1\}$, where $\{0,1\}$ is the set of values 0 and 1 rather than an interval and $\mathrm{B}(\mathrm{x})=1$ or 0 indicates whether element $\mathrm{x}$ in $\mathrm{U}$ is a member of the set $\mathrm{B}$ or not.

\subsection{Linguistic variable}

A linguistic variable is some fuzzy set joining values sharing some common property, usually familiar to human beings. For example, if we consider performance of a supplier, then high could be a linguistic variable. A set of linguistic variables is used to represent the original domain set in terms of the variables.

\subsection{Fuzzy numbers}

Fuzzy numbers are uncertain numbers for which, in addition to knowing a range of possible values, one can say that some values are more plausible or 'more possible' than others.

\subsection{Fuzzy Triangular Numbers}

A is a fuzzy number if A is normal and convex fuzzy set of $\mathrm{U}$. Triangular fuzzy number $A=\left(a_{1}, a_{2}, a_{3}\right)$ where $a_{2}$ is the value where the membership function of the fuzzy numberis1.0, $a_{1}$ is the left distribution and $a_{3}$ is the right distribution of the confidence interval of the fuzzy number A. The membership function is defined as

$$
\begin{aligned}
\mu_{A}(x) & =\left(x-a_{1}\right) /\left(a_{2}-a_{1}\right), \quad a_{1}<x \leq a_{2} \\
& =\left(a_{3}-x\right) /\left(a_{3}-a_{2}\right), \quad a_{2}<x \leq a_{3} \\
& =0, \text { otherwise }
\end{aligned}
$$

The triangular fuzzy number is based on a three-value judgment: the minimum possible value $a_{1}$, the most possible value $\mathrm{a}_{2}$ and the maximum possible value $\mathrm{a}_{3}$.

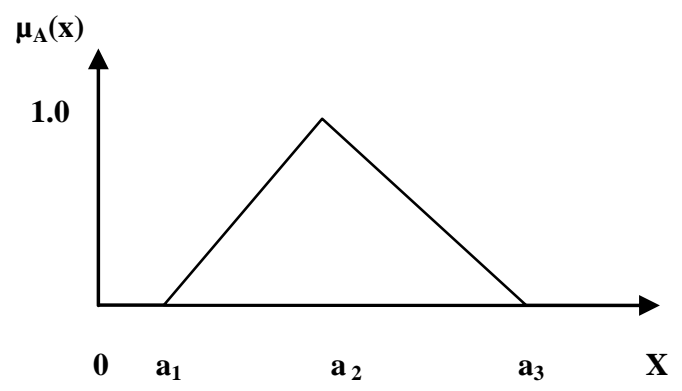

Figure 1 Triangular fuzzy number $A=\left(a_{1}, a_{2}, a_{3}\right)$

Let $A$ and $B$ be two fuzzy numbers parameterized by the triplet $\left(a_{1}, a_{2}, a_{3}\right)$ and $\left(b_{1}, b_{2}, b_{3}\right)$ respectively. Then the operations of triangular fuzzy numbers are expressed as:

$$
\begin{aligned}
A \oplus B & =\left(a_{1}, a_{2}, a_{3}\right) \oplus\left(b_{1}, b_{2}, b_{3}\right) \\
& =\left(a_{1}+b_{1}, a_{2}+b_{3}, a_{3}+b_{3}\right), \\
A \Theta B & =\left(a_{1}, a_{2}, a_{3}\right) \Theta\left(b_{1}, b_{2}, b_{3}\right) \\
& =\left(a_{1}-b_{3}, a_{2}-b_{2}, a_{3}-b_{1}\right), \\
A \otimes B & =\left(a_{1}, a_{2}, a_{3}\right) \otimes\left(b_{1}, b_{2}, b_{3}\right) \\
& =\left(a_{1} \times b_{1}, a_{2} \times b_{2}, a_{3} \times b_{3}\right), \\
A \emptyset \dot{B} & =\left(a_{1}, a_{2}, a_{3}\right) \varnothing\left(b_{1}, b_{2}, b_{3}\right) \\
& =\left(a_{1} / b_{3}, a_{2} / b_{2}, a_{3} / b_{1}\right) .
\end{aligned}
$$

\subsection{Defuzzification}

The defuzzification and mapping of the fuzzy number $\mathrm{A}=$ $\left(a_{1}, a_{2}, a_{3}\right)$ value in to a real numbers, the method of centre of gravity is used as follows

Defuzzy $A=a_{1}+\left[\left(a_{3}-a_{1}\right)+\left(a_{2}-a_{1}\right)\right] / 3$

\section{Basic Model}

The characteristic of all multi - criteria problems is to select the most appropriate action from various available alternatives. Different organizations select their optimal supplier using the fuzzy model described below. Fuzzy mathematical model includes the following steps.

Step 1 Determining the various criteria.

Step 2 Defining the set of linguistic variables.

Step 3 Identifying weights of the criteria.

Step 4 Identifying weights of the Decision makers. 
Step5 Presenting the decision makers preferences each supplier in terms of linguistic terms like: Very Poor, Poor, Fair, ... Good, Very Good, Excellent.

Step 6 Evaluating the supplier the supplier using linguistic variables.

Step 7 Constructing the fuzzy decision matrix using TFN's obtained through linguistic evaluation

$\widetilde{D}=\left(\begin{array}{ccccc}\widetilde{x}_{11} & \widetilde{x}_{12} & \ldots \ldots \ldots & \widetilde{x}_{1 n} \\ \widetilde{x}_{21} & \widetilde{x}_{22} & \ldots \ldots \ldots & \widetilde{x}_{2 n} \\ \cdot & \cdot & & \\ \cdot & \dot{\tilde{x}}_{m 2} & \ldots \ldots \ldots & \cdot \\ \widetilde{x}_{m 1} & \ldots \ldots & \cdot\end{array}\right)$

where $\tilde{x}_{i j}=\left(a_{i j}, b_{i j}, c_{i j}\right)$ and $\widetilde{w}_{j}=\left(w_{j 1}, w_{j 2}, w_{j 3}\right) \quad$ are weights of each criterion.

Step 8 Constructing the normalized fuzzy matrix as in (Wang 2009) using

$$
\begin{array}{r}
\tilde{r}_{i j}=\left(\frac{a_{i j}}{c_{j}^{*}}, \frac{b_{i j}}{c_{j}^{*}}, \frac{c_{i j}}{c_{j}^{*}}\right), i=1,2, \ldots m, j \in B \\
\tilde{r}_{i j}=\left(\frac{\overline{\overline{a_{J}}}}{c_{i j}}, \frac{\overline{a_{J}}}{b_{i j}}, \frac{\overline{a_{J}}}{a_{i j}}\right), i=1,2, \ldots m, j \in C
\end{array}
$$

where $B=$ set of benefit criteria, $C=$ set of cost criteria

$$
c_{j}^{*}=\max _{i} c_{i j}, j \in B, \overline{a_{J}}=\min _{i} c_{i j}, j \in C
$$

Step 9 Constructing the defuzzified decision matrix for defuzzification and mapping of the fuzzy number $\tilde{x}_{i j}=$ $\left(a_{i j}, b_{i j}, c_{i j}\right)$ in to real number, the centre of gravity method [9] is used here defuzzy $\tilde{x}_{i j}=\left[\left(c_{i j-} a_{i j}\right)+\right.$ $\left.\left(b_{i j}-a_{i j}\right)\right] 3^{-1}+a_{i j}$

Step 10 Constructing the rank matrices $R_{j}$ where the rows of the matrix are the suppliers and columns are DM's opinion based on $\mathrm{j}^{\text {th }}$ criteria. For each criteria rank matrices are obtained as

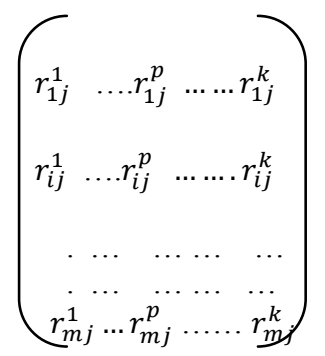

where $\mathrm{i}=1,2,3, \ldots \ldots \mathrm{m}, \mathrm{j}=1,2,3, \ldots \ldots \mathrm{n}$ and

$\mathrm{p}=1,2,3, \ldots \ldots \mathrm{k}$

Step 11 Computing the linear sum $\sum_{p=1}^{k} r_{i j}^{p} \quad$ of each row of rank matrix for all the DM's

Step 12 Constructing the final grade matrix $\mathrm{R}_{\mathrm{G}}$ taking suppliers along rows and criteria along columns

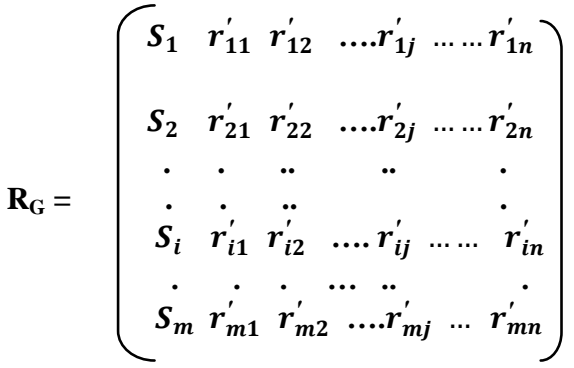

Step 13 Converting the $\mathrm{R}_{\mathrm{G}}$ matrix in to Preference matrix using Schwartz Sequential Dropping SSD method (also called Beat Path Method) [10].

\section{Procedure for SSD method:}

Let $d[A, B]$ be the number of criteria which prefer Supplier A to Supplier B. Calculate the strength $p[A, B]$ of the strongest path from A to B.

Supplier A is a potential supplier if and only if $p[A, B] \geq$ $\mathrm{p}[\mathrm{B}, \mathrm{A}]$ for every other Supplier B.

- Construct the matrix of pair wise defeats

- Calculate the strongest path for each pair of suppliers

- Calculate the strengths of the strongest paths and identify the potential supplier

Step 14 Ranking of suppliers can be made by multiplying the strongest path matrix with the corresponding weight vector of criteria. Suppliers are ranked considering highest value as the first rank and the lowest as the last rank.

\section{Illustrative Example of Supplier Selection Process \\ A Case Study}

Asia Energy Corporation-Technologies Unlimited (AEC) is a small scale industry located in Chennai, India. AEC is an house of Reverse Osmosis Water Treatment solutions. It manufactures electronic dosing pumps, pressure sand/carbon filters in stainless steel, micron filters, ozone generators, etc. For its unit it procures raw materials from various vendors. The supplier selection is made by the two partners and the Plant Engineer. The supplier selection is made based on criteria: Cost of material, Product Quality, Availability (Long Term) of product, Promptness in delivery, Service Quality, After sales Guarantee, Payment Terms. In our study we have focused on four criteria only.

Suppose that the company wishes to select a supplier. After initial screening four suppliers $S_{1}, S_{2}, S_{3}, S_{4}$ are selected for further evaluation. A committee of three decision makers/experts $D_{1}, D_{2}$ and $D_{3}$ rank the above suppliers and select the most appropriate supplier. The proposed method enhances the decision making by the group based on Schulze method. The method is described in the following steps.

Step 1:

Four benefit criteria obtained from expert opinions, namely Product Quality $\mathrm{C}_{1}$, Service Quality $\mathrm{C}_{2}$, Availability (Long Term) of product $\mathrm{C}_{3}$ and Promptness in Supply $\mathrm{C}_{4}$ are considered for selection. Step 2:

The three DM's use linguistic variables (table 1) to rate the importance of suppliers ( table2 ) 
Table 1. The Linguistic Importance Scale Of Suppliers

\begin{tabular}{|lr|c|}
\hline $\begin{array}{l}\text { Linguistic expressions for } \\
\text { importance }\end{array}$ & \multicolumn{1}{l|}{$\begin{array}{l}\text { Fuzzy } \\
\text { importance } \\
\text { scale }\end{array}$} \\
\hline Very Poor & VP & $(\mathbf{0 , 0 , 1 )}$ \\
\hline Poor & P & $(\mathbf{0 , 1 , 3 )}$ \\
\hline Fair & F & $(\mathbf{1 , 3 , 5 )}$ \\
\hline Medium Good & MG & $(\mathbf{3 , 5 , 7 )}$ \\
\hline Good & G & $(\mathbf{5 , 7 , 9 )}$ \\
\hline Very Good & VG & $\mathbf{( 7 , 9 , 1 0 )}$ \\
\hline Excellent & E & $(\mathbf{9 , 1 0 , 1 0 )}$ \\
\hline
\end{tabular}

Table 2. Suppliers' Performance Ratings With Respect To The Selection Criteria

\begin{tabular}{|c|c|c|c|c|c|c|c|c|}
\hline & \multicolumn{4}{|c|}{ C1 } & \multicolumn{4}{|c|}{ C2 } \\
\hline & S1 & $\mathbf{S 2}$ & S3 & S4 & S1 & S2 & S3 & S4 \\
\hline D1 & $\mathrm{G}$ & $\mathbf{E}$ & $\mathbf{G}$ & VG & $\mathbf{G}$ & VG & $\mathbf{E}$ & VG \\
\hline D2 & $\mathrm{G}$ & $\mathrm{E}$ & $\mathrm{G}$ & VG & $\mathrm{G}$ & VG & $\mathrm{E}$ & VG \\
\hline D3 & $\mathbf{G}$ & $\mathbf{E}$ & VG & VG & $\mathbf{G}$ & VG & $\mathbf{E}$ & $\mathbf{G}$ \\
\hline
\end{tabular}

\begin{tabular}{|c|c|c|c|cc|c|c|c|c|}
\hline & \multicolumn{4}{|c|}{ C3 } & \multicolumn{4}{c|}{ C4 } \\
& S1 & S2 & S3 & S4 & S1 & S2 & S3 & S4 \\
\hline D1 & VG & VG & G & VG & VG & VG & VG & VG \\
\hline D2 & VG & E & G & VG & VG & E & VG & VG \\
\hline D3 & VG & E & G & VG & VG & VG & VG & E \\
\hline
\end{tabular}

Table 5. Fuzzy Decision Matrix and Criteria Weights

\begin{tabular}{|c|c|c|c|c|c|}
\hline & & C1 & $\mathrm{C2}$ & $\mathrm{C3}$ & C4 \\
\hline \multirow{5}{*}{ D1 } & $\mathbf{S}$ & $(5,7,9)$ & $(5,7,9)$ & $(7,9,10)$ & $(7,9,10)$ \\
\hline & $\frac{1}{\mathrm{c}}$ & & & & \\
\hline & $\begin{array}{l}S \\
2\end{array}$ & $(\mathbf{9 , 1 0 , 1 0 )}$ & $(7,9,10)$ & $(7,9,10)$ & $(7,9,10)$ \\
\hline & $\begin{array}{l}S \\
3\end{array}$ & $(5,7,9)$ & $(9,10,10)$ & $(5,7,9)$ & $(7,9,10)$ \\
\hline & $\begin{array}{l} \\
4\end{array}$ & $\begin{array}{l}(7,9,10) \\
\end{array}$ & $\begin{array}{l}(7,9,10) \\
\end{array}$ & $(7,9,10)$ & $(7,9,10)$ \\
\hline \multirow{4}{*}{ D2 } & $\begin{array}{l} \\
1\end{array}$ & $(5,7,9)$ & $(5,7,9)$ & $(7,9,10)$ & $(\mathbf{7 , 9 , 1 0 )}$ \\
\hline & $\begin{array}{l}\mathbf{S} \\
2\end{array}$ & $(9,10,10)$ & $(7,9,10)$ & $\begin{array}{c}(9,10,10 \\
)\end{array}$ & $(9,10,10)$ \\
\hline & $\begin{array}{l}\mathbf{S} \\
\mathbf{3}\end{array}$ & $(5,7,9)$ & $(9,10,10)$ & $(5,7,9)$ & $(\mathbf{7 , 9 , 1 0 )}$ \\
\hline & $\begin{array}{l}S \\
4 \\
\end{array}$ & $(7,9,10)$ & $(7,9,10)$ & $(7,9,10)$ & $(7,9,10)$ \\
\hline \multirow[t]{4}{*}{ D3 } & $\begin{array}{l}\mathrm{S} \\
\mathbf{1} \\
\end{array}$ & $(5,7,9)$ & $(5,7,9)$ & $\begin{array}{l}(7,9,10) \\
\end{array}$ & $(7,9,10)$ \\
\hline & $\begin{array}{l} \\
2 \\
\end{array}$ & $(9,10,10)$ & $(7,9,10)$ & $\begin{array}{c}(9,10,10 \\
)\end{array}$ & $(7,9,10)$ \\
\hline & $\begin{array}{l}\mathbf{S} \\
\mathbf{3} \\
\end{array}$ & $(7,9,10)$ & $(9,10,10)$ & $(5,7,9)$ & $(\mathbf{7 , 9 , 1 0 )}$ \\
\hline & $\begin{array}{l} \\
4 \\
\end{array}$ & $(7,9,10)$ & $(5,7,9)$ & $(7,9,10)$ & $(9,10,10)$ \\
\hline $\begin{array}{c}\text { Weight } \\
\text { s }\end{array}$ & & $\begin{array}{c}(0.7,0.9,1 \\
)\end{array}$ & $\begin{array}{c}(\mathbf{0 . 8 3}, 0.96,1 \\
)\end{array}$ & $(0.9,1,1)$ & $\begin{array}{c}(0.77,0.93,1 \\
)\end{array}$ \\
\hline $\begin{array}{c}\text { Weight } \\
\text { s }\end{array}$ & & 0.24 & 0.25 & 0.26 & 0.25 \\
\hline
\end{tabular}

International Journal of Computer Applications (0975 - 8887)

Volume 54-No.7, September 2012

Step 3:

The linguistic variables shown in table 3 is used to assess the importance of criteria (table 4).

Table 3. The Linguistic Performance Scale Of Criteria

\begin{tabular}{|lr|c|}
\hline \multicolumn{2}{|c|}{$\begin{array}{c}\text { inguistic expressions for } \\
\text { performance }\end{array}$} & $\begin{array}{c}\text { Fuzzy } \\
\text { Performance } \\
\text { scale }\end{array}$ \\
\hline Low & L & $(\mathbf{0 , 0 , 0 . 1 )}$ \\
\hline Medium Low & ML & $(\mathbf{0 , 0 . 1 , 0 . 3 )}$ \\
\hline Medium & M & $(\mathbf{0 . 1 , 0 . 3 , 0 . 5 )}$ \\
\hline Medium High & MH & $(\mathbf{0 . 3 , 0 . 5 , 0 . 7 )}$ \\
\hline High & H & $(\mathbf{0 . 5 , 0 . 7 , 0 . 9 )}$ \\
\hline Very High & VH & $(\mathbf{0 . 7 , 0 . 9 , 1 . 0 )}$ \\
\hline Commendable & C & $(\mathbf{0 . 9 , 1 . 0 , 1 . 0 )}$ \\
\hline
\end{tabular}

Table 4.Weight Importance Of Criteria

\begin{tabular}{|c|c|c|c|}
\hline \multirow{2}{*}{ Criteria } & \multicolumn{3}{|c|}{ Decision maker } \\
\cline { 2 - 4 } & $\mathbf{D}_{\mathbf{1}}$ & $\mathbf{D}_{\mathbf{2}}$ & $\mathbf{D}_{\mathbf{3}}$ \\
\hline $\mathbf{C}_{\mathbf{1}}$ & $\mathbf{V H}$ & $\mathbf{V H}$ & $\mathbf{V H}$ \\
\hline $\mathbf{C}_{\mathbf{2}}$ & $\mathbf{C}$ & $\mathbf{C}$ & $\mathbf{C}$ \\
\hline $\mathbf{C}_{\mathbf{3}}$ & $\mathbf{C}$ & $\mathbf{C}$ & $\mathbf{C}$ \\
\hline $\mathbf{C}_{\mathbf{4}}$ & $\mathbf{V H}$ & $\mathbf{V H}$ & $\mathbf{C}$ \\
\hline
\end{tabular}

Step 4:

Construct the fuzzy decision matrix as shown in table 5 using TFNs and determine the weight of each criteria based on table 3 and 4

Figure1: Membership Function of Triangular fuzzy Numbers corresponding to linguistic scale

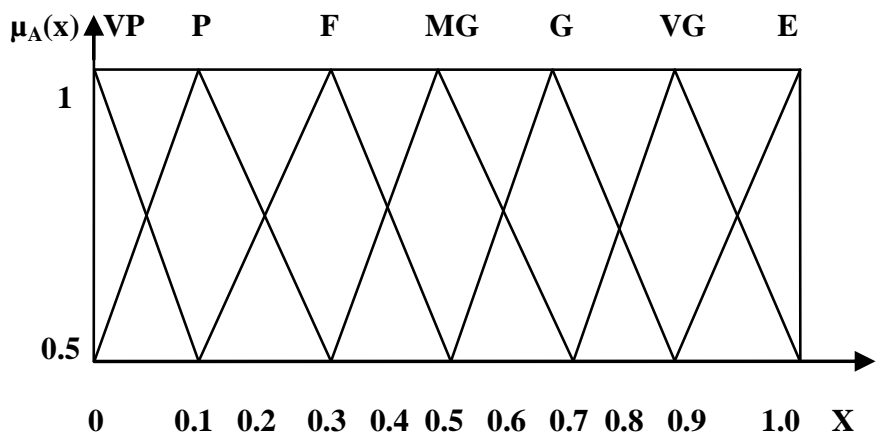

Step 5:

Construct the normalized fuzzy decision matrix as shown in table 6 using step 7 mentioned in Section 4. 
Table 6: Fuzzy Normalized Decision matrix

\begin{tabular}{|c|c|c|c|c|c|}
\hline & & C1 & $\mathrm{C2}$ & C3 & $\mathrm{C4}$ \\
\hline \multirow[t]{4}{*}{ D1 } & S1 & $(0.5,0.7,0.9)$ & $(0.5,0.7,0.9)$ & $(0.7,0.9,1)$ & $(0.7,0.9,1)$ \\
\hline & S2 & $(0.9,1,1)$ & $(0.7,0.9,1)$ & $\begin{array}{l}(0.7,0.9,1) \\
\end{array}$ & $(0.7,0.9,1)$ \\
\hline & S3 & $(0.5,0.7,0.9)$ & $(0.9,1,1)$ & $(0.5,0.7,0.9)$ & $(0.7,0.9,1)$ \\
\hline & S4 & $(0.7,0.9,1)$ & $(0.7,0.9,1)$ & $(0.7,0.9,1)$ & $(0.7,0.9,1)$ \\
\hline \multirow[t]{4}{*}{ D2 } & S1 & $(0.5,0.7,0.9)$ & $(0.5,0.7,0.9)$ & $\begin{array}{l}(0.7,0.9,1) \\
\end{array}$ & $(0.7,0.9,1)$ \\
\hline & S2 & $(0.9,1,1)$ & $\begin{array}{l}(0.7,0.9,1) \\
\end{array}$ & $\begin{array}{l}(0.9,1,1) \\
\end{array}$ & $\begin{array}{l}(0.9,1,1) \\
\end{array}$ \\
\hline & S3 & $(0.5,0.7,0.9)$ & $(0.9,1,1)$ & $(0.5,0.7,0.9)$ & $(0.7,0.9,1)$ \\
\hline & S4 & $(0.7,0.9,1)$ & $(0.7,0.9,1)$ & $(0.7,0.9,1)$ & $(0.7,0.9,1)$ \\
\hline \multirow[t]{4}{*}{ D3 } & S1 & $(0.5,0.7,0.9)$ & $(0.5,0.7,0.9)$ & $(0.7,0.9,1)$ & $(0.7,0.9,1)$ \\
\hline & S2 & $(0.9,1,1)$ & $(0.7,0.9,1)$ & $(0.9,1,1)$ & $(0.7,0.9,1)$ \\
\hline & S3 & $(0.7,0.9,1)$ & $(0.9,1,1)$ & $(0.5,0.7,0.9)$ & $(0.7,0.9,1)$ \\
\hline & S4 & $(0.7,0.9,1)$ & $(0.5,0.7,0.9)$ & $(0.7,0.9,1)$ & $(0.9,1,1)$ \\
\hline
\end{tabular}

Step 6:

Construct the defuzzified decision matrix as shown in table 7 using method mention in equation (2).

Table 7:The defuzzified Decision matrix

\begin{tabular}{|c|c|c|c|c|c|}
\hline & & C1 & C2 & C3 & C4 \\
\hline D1 & S1 & 0.7 & 0.7 & 0.87 & 0.87 \\
\cline { 2 - 6 } & S2 & $\mathbf{0 . 9 7}$ & $\mathbf{0 . 8 7}$ & $\mathbf{0 . 8 7}$ & $\mathbf{0 . 8 7}$ \\
\cline { 2 - 6 } & S3 & 0.7 & $\mathbf{0 . 9 7}$ & $\mathbf{0 . 7}$ & $\mathbf{0 . 8 7}$ \\
\cline { 2 - 5 } & S4 & $\mathbf{0 . 8 7}$ & $\mathbf{0 . 8 7}$ & $\mathbf{0 . 8 7}$ & $\mathbf{0 . 8 7}$ \\
\hline D2 & S1 & $\mathbf{0 . 7}$ & $\mathbf{0 . 7}$ & $\mathbf{0 . 8 7}$ & $\mathbf{0 . 8 7}$ \\
\cline { 2 - 5 } & S2 & $\mathbf{0 . 9 7}$ & $\mathbf{0 . 8 7}$ & $\mathbf{0 . 9 7}$ & $\mathbf{0 . 9 7}$ \\
\cline { 2 - 5 } & S3 & $\mathbf{0 . 7}$ & $\mathbf{0 . 9 7}$ & $\mathbf{0 . 7}$ & $\mathbf{0 . 8 7}$ \\
\cline { 2 - 5 } & S4 & $\mathbf{0 . 8 9}$ & $\mathbf{0 . 8 7}$ & $\mathbf{0 . 8 7}$ & $\mathbf{0 . 8 7}$ \\
\hline D3 & S1 & $\mathbf{0 . 7}$ & $\mathbf{0 . 7}$ & $\mathbf{0 . 8 7}$ & $\mathbf{0 . 8 7}$ \\
\cline { 2 - 5 } & S2 & $\mathbf{0 . 9 7}$ & $\mathbf{0 . 8 7}$ & $\mathbf{0 . 9 7}$ & $\mathbf{0 . 8 7}$ \\
\cline { 2 - 5 } & S3 & $\mathbf{0 . 8 7}$ & $\mathbf{0 . 9 7}$ & $\mathbf{0 . 7}$ & $\mathbf{0 . 8 7}$ \\
\cline { 2 - 5 } & S4 & $\mathbf{0 . 8 7}$ & $\mathbf{0 . 7}$ & $\mathbf{0 . 8 7}$ & $\mathbf{0 . 9 7}$ \\
\hline
\end{tabular}

Step 7:

Construct $\mathrm{R}_{\mathrm{j}}$ matrix taking into an account the proper $\mathrm{DM}$ weights of every DM as shown in table 8 and Table $8 \mathrm{a}$. (here weights are calculated using conventional method [11]).

Table 8. The Rank Matrix $\mathbf{R}_{\mathrm{j}}$

\begin{tabular}{|c|c|c|c|c|c|}
\hline C1 & D1 & D2 & D3 & $\sum$ & RANK \\
\hline S1 & 0.175 & 0.175 & 0.175 & 0.525 & 4 \\
\hline S2 & $\mathbf{0 . 2 4 2 5}$ & $\mathbf{0 . 2 4 2 5}$ & $\mathbf{0 . 2 4 2 5}$ & $\mathbf{0 . 7 2 7 5}$ & 1 \\
\hline S3 & $\mathbf{0 . 1 7 5}$ & $\mathbf{0 . 1 7 5}$ & $\mathbf{0 . 2 1 7 5}$ & $\mathbf{0 . 5 6 7 5}$ & 3 \\
\hline S4 & $\mathbf{0 . 2 1 7 5}$ & $\mathbf{0 . 2 1 7 5}$ & $\mathbf{0 . 2 1 7 5}$ & $\mathbf{0 . 6 5 2 5}$ & 2 \\
\hline
\end{tabular}

\begin{tabular}{|c|c|c|c|c|c|}
\hline C2 & D1 & D2 & D3 & $\sum$ & RANK \\
\hline S1 & 0.175 & 0.175 & 0.175 & 0.525 & 4 \\
\hline S2 & 0.2175 & $\mathbf{0 . 2 1 7 5}$ & $\mathbf{0 . 2 1 7 5}$ & $\mathbf{0 . 6 5 2 5}$ & 2 \\
\hline S3 & $\mathbf{0 . 2 4 2 5}$ & $\mathbf{0 . 2 4 2 5}$ & $\mathbf{0 . 2 4 2 5}$ & $\mathbf{0 . 7 2 7 5}$ & 1 \\
\hline S4 & $\mathbf{0 . 2 1 7 5}$ & $\mathbf{0 . 2 1 7 5}$ & $\mathbf{0 . 1 7 5}$ & $\mathbf{0 . 6 1}$ & 3 \\
\hline
\end{tabular}

Table 8a: The Rank Matrix $\mathbf{R}_{\mathbf{j}}$

\begin{tabular}{|l|c|c|c|c|c|}
\hline C3 & D1 & D2 & D3 & $\sum$ & RANK \\
\hline S1 & 0.2175 & 0.2175 & 0.2175 & 0.6525 & 2 \\
\hline S2 & 0.2175 & 0.2425 & 0.2425 & 0.7025 & 1 \\
\hline S3 & 0.175 & 0.175 & 0.175 & 0.525 & 4 \\
\hline S4 & 0.2175 & 0.2175 & 0.2175 & 0.6525 & 2 \\
\hline \multicolumn{7}{|l|}{} \\
\hline C4 & D1 & D2 & D3 & $\sum$ & RANK \\
\hline S1 & 0.2175 & 0.2175 & 0.2175 & 0.6525 & 3 \\
\hline S2 & 0.2175 & 0.2425 & 0.2175 & 0.6775 & 1 \\
\hline S3 & 0.2175 & 0.2175 & 0.2175 & 0.6525 & 3 \\
\hline S4 & 0.2175 & 0.2175 & 0.2425 & 0.6775 & 1 \\
\hline
\end{tabular}

Step 8:

The linear sum of ranks of each row for all the DM's are calculated as shown in tables 8 and $8 \mathrm{a}$ and final rank of all suppliers by decision makers is also calculated.

Step 9:

The final grade matrix $\mathrm{R}_{\mathrm{G}}$ of all suppliers is calculated as

$$
\mathbf{R}_{\mathrm{G}}=\left(\begin{array}{cccc}
4 & 1 & 3 & 2 \\
4 & 2 & 1 & 3 \\
2 & 1 & 4 & 2 \\
3 & 1 & 3 & 1
\end{array}\right)
$$

Step 10:

Preference of suppliers based on four criteria calculated from $R_{G}$ matrix is as follows

$$
\begin{array}{lllll}
1 & S_{2} & S_{4} & S_{3} & S_{1} \\
1 & S_{3} & S_{2} & S_{4} & S_{1} \\
1 & S_{2} & S_{1} & S_{4} & S_{3} \\
1 & S_{2} & S_{4} & S_{1} & S_{3}
\end{array}
$$

Next the matrix of pair wise defeat looks as in table 9 
Table 9. The Matrix Of Pairwise Defeat

\begin{tabular}{|c|c|c|c|c|}
\hline & $\mathbf{p}\left[{ }^{*}, \mathbf{S 1}\right]$ & $\mathbf{p}\left[{ }^{*}, \mathbf{S 2}\right.$ & $\mathbf{p}\left[{ }^{*}, \mathbf{S 3}\right]$ & $\mathbf{p}\left[{ }^{*}, \mathbf{S 4}\right]$ \\
\hline $\mathbf{p}\left[{ }^{*}, \mathbf{S 1}\right]$ & - & $\mathbf{0}$ & $\mathbf{2}$ & $\mathbf{0}$ \\
\hline $\mathbf{p}\left[{ }^{*}, \mathbf{S 2}\right]$ & $\mathbf{4}$ & - & $\mathbf{3}$ & $\mathbf{4}$ \\
\hline $\mathbf{p}\left[{ }^{*}, \mathbf{S 3}\right]$ & $\mathbf{0}$ & $\mathbf{0}$ & - & $\mathbf{0}$ \\
\hline $\mathbf{p}\left[{ }^{*}, \mathbf{S} 4\right]$ & $\mathbf{3}$ & $\mathbf{0}$ & $\mathbf{3}$ & - \\
\hline
\end{tabular}

The graph of pair wise defeat is as follows

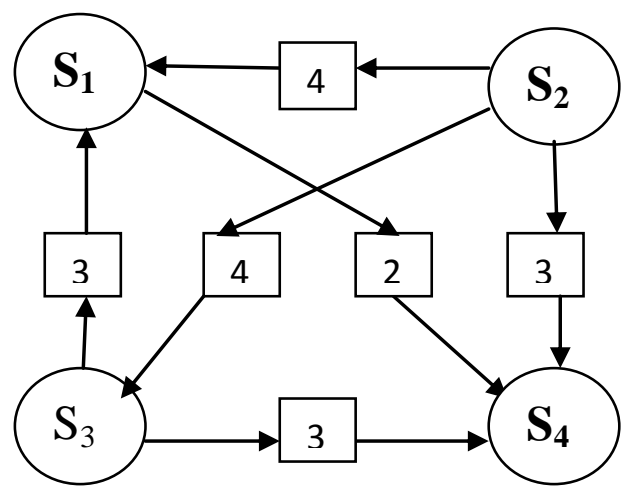

The strengths of the strongest paths are calculated as in table 10.

Table 10. The Strength Of The Strongest Paths

\begin{tabular}{|c|c|c|c|c|}
\hline & $\mathrm{d}[*, \mathrm{~S}]$ & $\mathbf{d}\left[{ }^{*}, \mathbf{S} 2\right]$ & $\mathrm{d}\left[{ }^{*}, \mathrm{~S} 1\right]$ & $\mathrm{d}\left[{ }^{*}, \mathrm{~S} 1\right]$ \\
\hline $\mathrm{d}\left[{ }^{*}, \mathrm{~S} 1\right]$ & - & 0 & 2 & 1 \\
\hline $\mathbf{d}[*, \mathbf{S 2}]$ & 4 & - & 3 & 4 \\
\hline d [*,S3] & 2 & 1 & - & 1 \\
\hline d [ [*,S4] & 3 & 0 & 3 & - \\
\hline
\end{tabular}

Here $\left[p\left(S_{2}, S_{1}\right)=4\right]>\left[p\left(S_{1}, S_{2}\right)=0\right], p\left(S_{2}, S_{4}\right)>p\left(S_{4}, S_{2}\right)$

$\mathrm{p}\left(\mathrm{S}_{2}, \mathrm{~S}_{3}\right)>\mathrm{p}\left(\mathrm{S}_{3}, \mathrm{~S}_{2}\right)$

Hence supplier $S_{2}$ is the optimal supplier.

Step 12:

Ranking of suppliers is made as follows

$\begin{array}{llllllc}\mathbf{0} & \mathbf{0} & \mathbf{2} & \mathbf{1} & \mathbf{0 . 2 4} & \mathbf{0 . 7 7} & \mathbf{4} \\ \mathbf{4} & \mathbf{0} & \mathbf{3} & \mathbf{4} & \mathbf{0 . 2 5} & \mathbf{2 . 7 5} & \mathbf{1} \\ \mathbf{2} & \mathbf{1} & \mathbf{0} & \mathbf{1} & \mathbf{0 . 2 6} & \mathbf{0 . 9 8} & \mathbf{3} \\ \mathbf{3} & \mathbf{0} & \mathbf{3} & \mathbf{0} & \mathbf{0 . 2 5} & \mathbf{1 . 5 0} & \mathbf{2}\end{array}$

The order of preference of the four suppliers are :

$$
\mathrm{S}_{2}>\mathrm{S}_{4}>\mathrm{S}_{3}>\mathrm{S}_{1}
$$

\section{Conclusion}

An enterprise can achieve maximum benefit or performance through proper selection of good suppliers. The process of supplier selection demands a systematic approach. This study of fuzzy group decision making frame work helps in effective evaluation of suppliers. In this paper we have ranked the suppliers using Schulze Beat Path method which is used in voting system. Here we have restricted our study to only four criteria. In case of extending it to more criteria, Floyd-Warshall algorithm can be used to calculate the strengths of the strongest paths. This algorithm can be used as it works by iteratively choosing a vertex on the graph as a 'waypoint.' If the path through the waypoint is shorter than the current shortest path the shortest path cost is updated. The above proposed approach is computationally simple and captures the uncertainty and imprecision in the group decision making process.

The subjectivity and vagueness can also be captured using Fuzzy Trapezoidal numbers unlike the TFN's used in this paper. This gives the direction for future research.

\section{References}

[1] R.E.Bellmann, L.A.Zadeh, :'Decision making in a fuzzy environment" management Sci.17 (1970).

[2] A Triangular Fuzzy Number-Based Approach to Group Decision Making with Linguistic Preference Information IEEE Conference Publications , 2008

[3] S.J.Chen and C.L.Hwang, Fuzzy Multiple Attribute decision-making, Methods and Applications

[4] Yoon, K. and Hwang, C. 1995, 'Multiple Attribute Decision Making: An Introduction', in Lewis-Beck, M. (Ed.), Quantitative Applications in the Social Sciences, Series/Number 07-104, Sage Publications.

[5] Kahraman, 2008). Fuzzy Multi-Criteria Decision Making: Theory and Applications with Recent Developments

[6] H.-J.Zimmermann, Fuzzy Sets,decision-making and Expert Systems, Kluwer Academic Publisher, Boston 1987

[7] J.C.Fodor and M.Roubens, Fuzzy Preference Modelling and Multi criteria Decision Support, Kluwer, Dordrecht, 1994.Lecture Notes in Economics and Mathematical Systems, vol. 375 (Springer, Heildelberg, 1993).

[8] M. Sakawa, Fuzzy Sets and Interactive Multi objective Optimization, Applied Information Technology, Plenum Press, New York 1993.

[9] Timothy Ross,Fuzzy Logic with Engineering Applications

[10] Schulze's beatpath voting method 1997 http://rangevoting.org/SchulzeExplan.html

[11] Deriving weights of criteria from inconsistent fuzzy comparison matrices by using the nearest weighted interval approx., Mohammad Izaduhah Hindawi Publishers. 